\title{
MODELLING AND SIMULATION OF A FLEXIBLE ENDOSCOPIC SURGICAL INSTRUMENT IN A TUBE
}

\author{
J.P. Khatait, M. Krijnen, \\ J.P. Meijaard, R.G.K.M. Aarts, D.M. Brouwer, J.L. Herder \\ Mechanical Automation \& Mechatronics \\ Faculty of Engineering Technology \\ University of Twente \\ P.O. Box 217, 7500 AE Enschede \\ The Netherlands
}

\begin{abstract}
Flexible instruments are increasingly used to carry out complex procedures in many surgical interventions. The instrument tip is remotely controlled by the surgeon. The inherent flexibility of the instrument, coupled with the friction inside the endoscope access channel and the convoluted shape of the endoscope inside the body, makes the control of the instrument tip difficult and complicated.

The objective of this paper is to model and to simulate a flexible endoscopic surgical instrument inside a tube and characterize its behaviour.

The surgical instrument is modelled as a series of interconnected beam elements. The endoscope channel is modelled as a rigid tube of uniform circular cross-section. A planar model of the flexible instrument with friction is considered in this paper. Normal reaction and friction forces are calculated at the nodes. A Stribeck based continuous friction model with increased friction at low velocity is implemented in the model. Simulations are carried out both for the insertion of the flexible instrument and for fine manipulation. SPACAR, an analysis tool for flexible multibody dynamic systems, has been used for the modelling and simulation.

The nodal displacement and force acting at the various nodes have been obtained depending on position and time. The
\end{abstract}

*Address all correspondence to this author. Tel.: +31 53489 5442. Fax: +31 53489 3631. Email: j.p.khatait@utwente.nl simulation for the fine tip manipulation shows the stick-slip behaviour and hysteresis. The simulation results show the effect of bending rigidity and friction on motion hysteresis.

\section{INTRODUCTION}

Surgical robotic systems are revolutionizing healthcare and medical services. Minimally-Invasive-Surgery (MIS), also termed as laparoscopic surgery, has greatly reduced the unnecessary damage to healthy tissues, leading to faster recovery, reduced infection rate, and reduced post-operative complications. The most of the limitations imposed by the conventional laparoscopic system are well addressed by the surgical robotic system by increasing dexterity, restoring proper hand-eye coordination and an ergonomic working position, and improving visualization. Furthermore, the ability of integrating and interfacing with various technologies has expanded the horizon of these robotic systems.

The state-of-the-art robotic surgery systems employ rigid instruments [1]. However, with conventional colonoscopy and with the emergence of Natural Orifice Transluminal Endoscopic Surgery (NOTES) and Single Incision Laparoscopic Surgery (SILS) procedures, the use of flexible instruments is inevitable. These flexible instruments are fed through access channels provided in the endoscope or endoscopic platform. The instrument tip is remotely controlled. The inherent flexibility of 


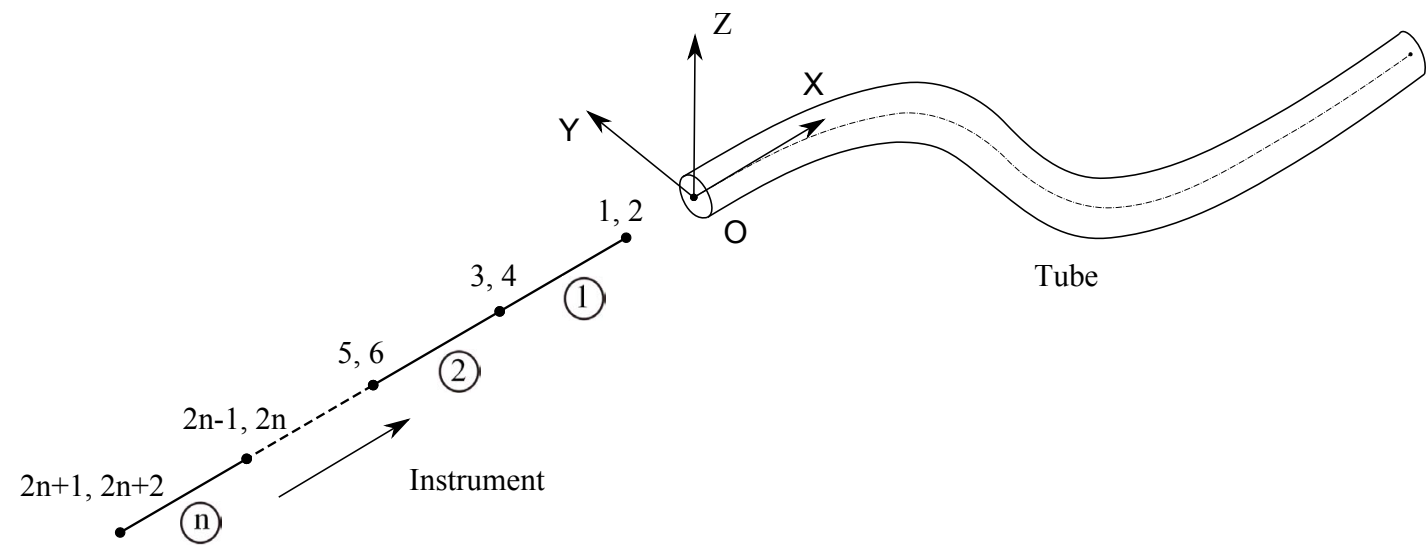

FIGURE 1. MODEL OF THE INSTRUMENT WITH THE TUBE AT THE BEGINNING OF INSERTION

the instrument, coupled with the friction inside the endoscope channel and the convoluted shape of the endoscope inside the body, makes the control of the instrument tip difficult and cumbersome. Furthermore, as the endoscopic procedures are becoming more invasive, the surgical instruments require complex manipulations. The instrument tip needs to deliver a certain amount of force or to orient in a particular way. The motion and force fidelity of these instruments is critical for achieving good surgical outcomes.

In endoscope-like surgical systems, the instrument is controlled from the proximal end. Nonlinearities are introduced in motion transmission by the friction forces between the instrument and the access channel. Moreover, the shape of the endoscope is not fixed. It changes depending on the location of the surgical site. There will be a change in the force/torque delivered which is dependent on the friction properties and the shape of the contacting surfaces. Since it is difficult to place the sensors at the distal end of the instrument, the actual position and the force delivered at the instrument tip are difficult to estimate and control. This makes the control of the instrument tip difficult and challenging.

The objective of this paper is to model and to simulate a flexible endoscopic surgical instrument inside a tube and characterize its behaviour. A tube represents the access channel provided in a typical endoscope for the flexible surgical instrument. A planar model of the flexible instrument with friction is considered in this paper. Ultimately, this model leads to the development of simpler models to use in overall system models and can possibly be used to improve the control and performance of the overall system. The characteristic behaviour of the flexible instrument translation can be analysed in a planar configuration.

In the next section, the planar model of the flexible instrument and contact model are explained. Simulation for the insertion and fine manipulation of the instrument are discussed thereafter. The effect of bending rigidity and friction is discussed in the subsequent section.

\section{MODELLING OF A FLEXIBLE ENDOSCOPIC SURGICAL INSTRUMENT}

A flexible multibody modelling approach has been used for the modelling of a flexible surgical instrument inside an endoscope channel. The surgical instrument is modelled as a series of interconnected two-noded beam elements. The endoscope channel is modelled as a rigid tube of uniform circular cross-section. The shape of the tube is defined by a centre line and the tube diameter. The contact between the beam and the tube is defined at the nodes, which are at the ends of the beam elements.

A computer program SPACAR [2] is used for the modelling and simulation of the flexible surgical instrument inside a tube. SPACAR is a modelling and simulation tool for multibody dynamic analysis of planar and spatial mechanisms and manipulators with flexible links.

The model of the flexible instrument together with the model of the tube is shown in Fig. 1. The origin of the global frame, O, is situated at the beginning of the tube and the initial tangential direction is the $\mathrm{X}$-axis. The encircled number, (n), represents the $n^{\text {th }}$ beam element. The translational nodal coordinates are represented by the odd numbers, while the even numbers denote the rotational nodal coordinates.

\section{Flexible Surgical Instrument as Flexible Beam}

The surgical instrument is modelled as a series of interconnected flexible beams elements, having one nodal point at the each end of the beam (Fig. 1). The planar beam element has two translational and one rotational coordinate defined at its nodes. The configuration of the beam element is described by the position vectors $\underline{x}^{p}$ and $\underline{x}^{q}$ of the end nodes $p$ and $q$ and 


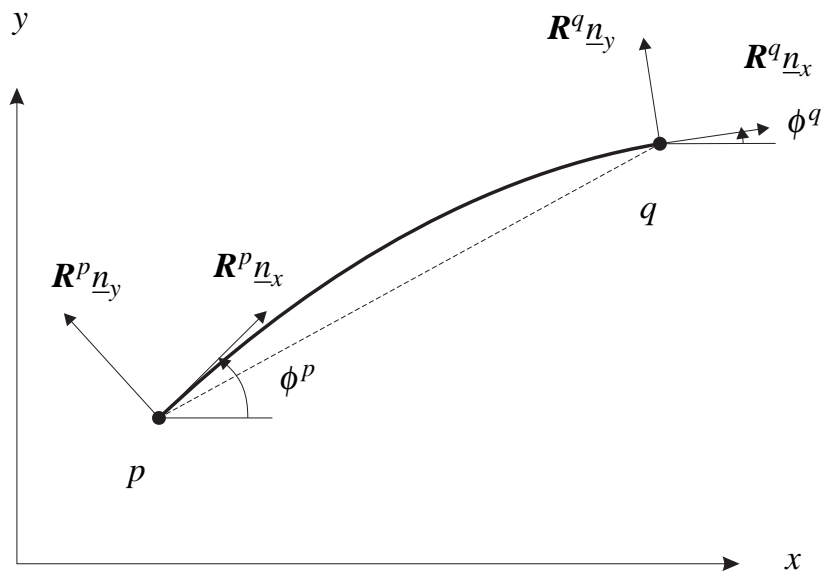

FIGURE 2. THE PLANAR FLEXIBLE BEAM ELEMENT

the angular orientation of the orthonormal triads $\left(\underline{n}_{x}, \underline{n}_{y}\right)$ rigidly attached to the element nodes [3,4], as shown in Fig. 2. The rotation part of the motion of the beam element is described by the planar rotation matrices $\boldsymbol{R}^{p}$ and $\boldsymbol{R}^{q}$, defined by

$$
\boldsymbol{R}^{p}=\left[\begin{array}{rr}
\cos \phi^{p} & -\sin \phi^{p} \\
\sin \phi^{p} & \cos \phi^{p}
\end{array}\right], \quad \boldsymbol{R}^{q}=\left[\begin{array}{rr}
\cos \phi^{q} & -\sin \phi^{q} \\
\sin \phi^{q} & \cos \phi^{q}
\end{array}\right]
$$

The nodal coordinates of the beam element are four Cartesian coordinates $\left(x^{p}, y^{p}\right),\left(x^{q}, y^{q}\right)$ describing the position of the beam in the $(x, y)$-coordinate system and two rotation angles $\phi^{p}$ and $\phi^{q}$ representing the angular orientation of the base vectors $\left(\boldsymbol{R}^{p} \underline{n}_{x}\right.$, $\left.\boldsymbol{R}^{p} \underline{n}_{y}\right)$ and $\left(\boldsymbol{R}^{q} \underline{n}_{x}, \boldsymbol{R}^{q} \underline{n}_{y}\right)$ at the nodes $p$ and $q$ respectively. Hence the vector of nodal coordinates is given by

$$
\underline{x}_{\text {beam }}=\left[x^{p}, y^{p}, \phi^{p} \mid x^{q}, y^{q}, \phi^{q}\right]^{T} .
$$

The beam element can account for the characteristic behaviour of the flexible instrument as can be understood from three deformation modes that are defined for the planar beam. The deformation $\varepsilon_{1}$ represents the elongation of the element, and the other two deformation modes, $\varepsilon_{2}$ and $\varepsilon_{3}$, are associated with the flexible deformation of the beam element. They are defined as:

$$
\begin{array}{ll}
\text { elongation: } & \varepsilon_{1}=l-l_{0}, \\
\text { bending: } & \varepsilon_{2}=-\left(\boldsymbol{R}^{p} \underline{n}_{y}, \underline{l}\right), \\
& \varepsilon_{3}=\left(\boldsymbol{R}^{q} \underline{n}_{y}, \underline{l}\right),
\end{array}
$$

where $l_{0}$ is the reference length of the element, $l$ is the length of the vector $\underline{l}$, and the vector $\underline{l}$ is defined by

$$
\underline{l}=\underline{x}^{q}-\underline{x}^{p}=\left[x^{q}-x^{p}, y^{q}-y^{p}\right]^{T} .
$$

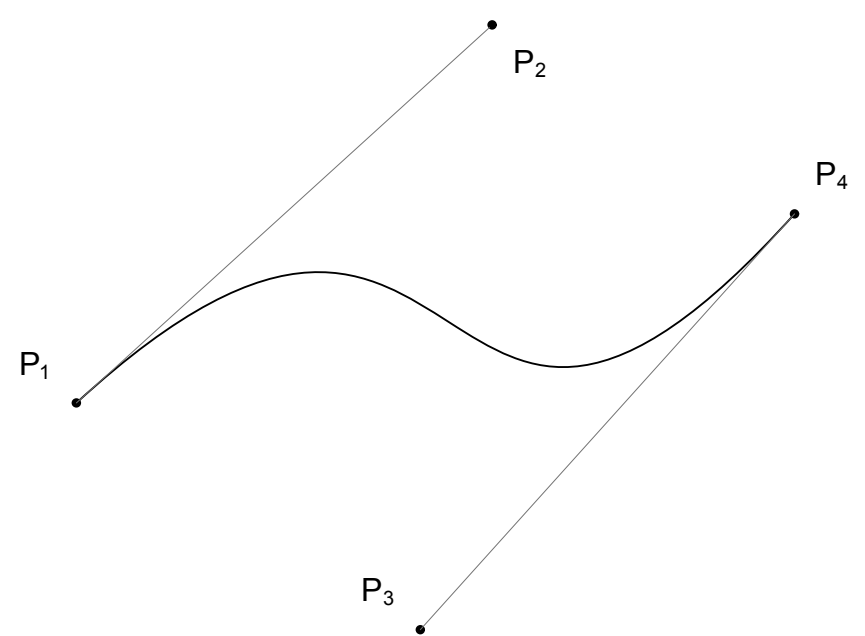

FIGURE 3. CUBIC BÉZIER CURVE DEFINED BY FOUR POINTS

To describe the complex shape of the complete instrument, multiple beam elements are connected. More beam elements are required to accurately model the instrument behaviour inside a tube with a complex shape. That also leads to high computation time required for the simulation. Therefore, an optimum number of elements are chosen so that most of the typical characteristics can be captured without unduly increasing the computation time. The curvature of the tube is reduced with respect to real anatomical features.

\section{Access Channel in The Endoscope as a Rigid Tube}

The access channel in the endoscope is modelled as a rigid tube. The shape of the tube is defined by a centre line. The centre line of the tube can be defined by a straight line, a circular arc, a Bézier curve, or a combination of these. Figure 3 illustrates a part of a tube defined by a Bézier curve. The curve defines the centre line of the tube. The first control point, $\mathbf{P}_{\mathbf{1}}$, and the last control point, $\mathbf{P}_{\mathbf{4}}$, define the end points of the curve. Intermediate control points, $\mathbf{P}_{\mathbf{2}}$ and $\mathbf{P}_{\mathbf{3}}$, influence the path of the curve. The first two and the last two control points define lines which are tangent to the beginning and the end of the curve [5]. Any point, $\mathbf{P}(u)=\left[\begin{array}{ll}x(u) & y(u)\end{array}\right]$, on a parametric cubic Bézier curve is given by

$$
\begin{array}{r}
\mathbf{P}(u)=(1-u)^{3} \mathbf{P}_{1}+3 u(1-u)^{2} \mathbf{P}_{2}+3 u^{2}(1-u) \mathbf{P}_{3}+u^{3} \mathbf{P}_{4} \\
=\left[\begin{array}{llll}
(1-u)^{3} & 3 u(1-u)^{2} & 3 u^{2}(1-u) & u^{3}
\end{array}\right]\left[\begin{array}{l}
\mathbf{P}_{1} \\
\mathbf{P}_{2} \\
\mathbf{P}_{3} \\
\mathbf{P}_{4}
\end{array}\right]
\end{array}
$$

where the parameter $0 \leq u \leq 1$, and the control points $\mathbf{P}_{1}, \mathbf{P}_{2}, \mathbf{P}_{3}$, and $\mathbf{P}_{4}$ define a Bézier polygon. Equation (5) can be written in 


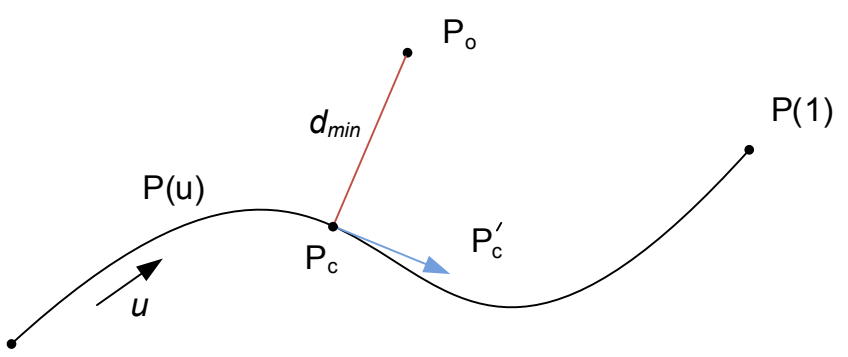

$\mathrm{P}(0)$

FIGURE 4. MINIMUM DISTANCE BETWEEN A POINT AND A CURVE

algebraic form as

$$
\begin{aligned}
\mathbf{P}(u) & =\mathbf{a}_{1}+\mathbf{a}_{2} u+\mathbf{a}_{3} u^{2}+\mathbf{a}_{4} u^{3} \\
& =\left[\begin{array}{llll}
1 & u & u^{2} & u^{3}
\end{array}\right]\left[\begin{array}{llll}
\mathbf{a}_{1} & \mathbf{a}_{2} & \mathbf{a}_{3} & \mathbf{a}_{4}
\end{array}\right]^{T} \\
& =\left[\begin{array}{llll}
1 & u & u^{2} & u^{3}
\end{array}\right]\left[\begin{array}{ll}
\mathbf{A}
\end{array}\right]
\end{aligned}
$$

where $\mathbf{a}_{i}$ is the vector-valued algebraic coefficients. From Eqn. (5) and Eqn. (6), A can be written as

$$
\mathbf{A}=\left[\begin{array}{l}
\mathbf{a}_{1} \\
\mathbf{a}_{2} \\
\mathbf{a}_{3} \\
\mathbf{a}_{4}
\end{array}\right]=\left[\begin{array}{l}
\mathbf{P}_{1} \\
-3 \mathbf{P}_{1}+3 \mathbf{P}_{2} \\
3 \mathbf{P}_{1}-6 \mathbf{P}_{2}+3 \mathbf{P}_{3} \\
-\mathbf{P}_{1}+3 \mathbf{P}_{2}-3 \mathbf{P}_{3}+\mathbf{P}_{4}
\end{array}\right]
$$

A tangent vector at any point on the curve is given by the parametric derivative of the curve. The parametric derivative of a cubic Bézier curve from Eqn. (6) is

$$
\mathbf{P}^{\prime}(u)=\left[\begin{array}{llll}
0 & 1 & 2 u & 3 u^{2}
\end{array}\right][\mathbf{A}]
$$

Normal from a point $\mathbf{P}_{0}$ to the centre line When the beam is in contact with the inner wall of the tube, the interaction force acts at the node. This requires calculation of the normal and tangent at the contact point. The minimum distance between a point $\mathbf{P}_{0}$ and a curve $\mathbf{P}(u)$ is the length of the line perpendicular to a tangent to the curve as shown in Fig. 4 . The problem is to find the value of $u$ which locates $\mathbf{P}_{c}$, the point on the curve where a line from $\mathbf{P}_{0}$ is perpendicular to the tangent line. The vector from $\mathbf{P}_{0}$ to the curve is $\left(\mathbf{P}_{c}-\mathbf{P}_{0}\right)$. The tangent vector $\mathbf{P}_{c}^{\prime}$ is given by the parametric derivative of the curve at point $\mathbf{P}_{c}$ (Eqn. (8)). For this vector and the tangent vector to be perpendicular,

$$
\left(\mathbf{P}_{c}-\mathbf{P}_{0}\right) . \mathbf{P}_{c}^{\prime}=0
$$

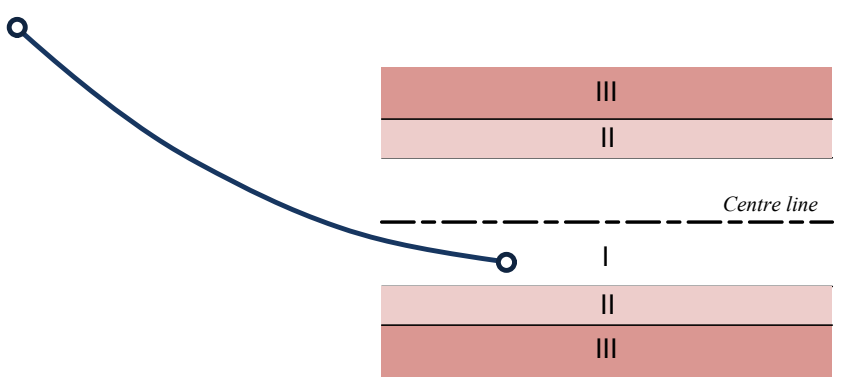

FIGURE 5. THREE REGIONS OF CONTACT

In terms of the algebraic coefficients describing $\mathbf{P}_{c}$ (Eqn. (6)), Eqn. (9) can be expanded to give

$$
c_{0}+c_{1} u+c_{2} u^{2}+c_{3} u^{3}+c_{4} u^{4}+c_{5} u^{5}=0
$$

where

$$
\begin{aligned}
& c_{0}=\mathbf{a}_{1} \cdot \mathbf{a}_{2}-\mathbf{P}_{0} \cdot \mathbf{a}_{2} \\
& c_{1}=2 \mathbf{a}_{1} \cdot \mathbf{a}_{3}+\mathbf{a}_{2} \cdot \mathbf{a}_{2}-2 \mathbf{P}_{0} \cdot \mathbf{a}_{3} \\
& c_{2}=3 \mathbf{a}_{1} \cdot \mathbf{a}_{4}+3 \mathbf{a}_{2} \cdot \mathbf{a}_{3}-3 \mathbf{P}_{0} \cdot \mathbf{a}_{4} \\
& c_{3}=4 \mathbf{a}_{2} \cdot \mathbf{a}_{4}+2 \mathbf{a}_{3} \cdot \mathbf{a}_{3} \\
& c_{4}=5 \mathbf{a}_{3} \cdot \mathbf{a}_{4} \\
& c_{5}=3 \mathbf{a}_{4} \cdot \mathbf{a}_{4}
\end{aligned}
$$

Equation (10) is solved, yielding one or more real roots, $0 \leq u \leq 1$, and $\mathbf{P}_{c}$ is evaluated. In order to have a unique solution, $\mathbf{P}_{0}$ should be such that $d_{\text {min }}$ is smaller than the minimum radius of curvature, $R_{\min }$. The minimum distance, $d_{\min }$, is given by

$$
d_{\text {min }}=\left|\mathbf{P}_{c}-\mathbf{P}_{o}\right|
$$

\section{Interaction of a Beam with the Inner Wall of a Tube}

The contact between the beam and the wall is defined at the nodes of the beam elements. As the node approaches the wall, the node experiences an equivalent normal force depending on the depth of penetration and the rate of penetration. Wall stiffness and damping are defined normal to the surface. Friction at the contact point is also defined. Therefore, depending on whether there is any sliding motion at the contact point or not, the node can experience a friction force in the tangential direction.

There are three contact regions defined depending on the position of the node (Fig. 5) [6]:

- Region I: No contact

- Region II: Transition

- Region III: Full contact 


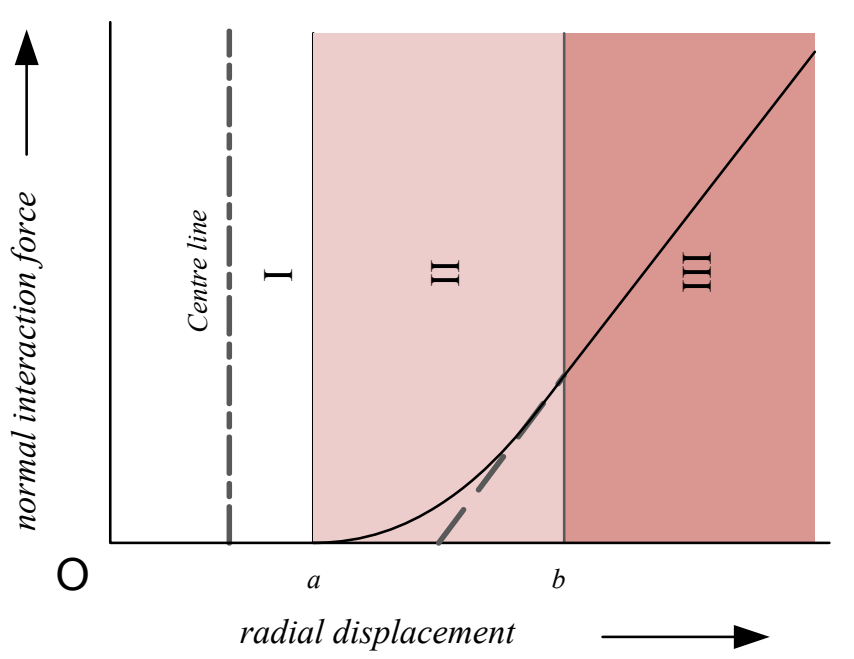

FIGURE 6. MODELLING OF CONTACT BETWEEN THE BEAM AND INNER WALL OF THE TUBE

Region I defines the zone when there is no contact at all. Region III is the zone where the beam is in full contact with the inner wall of the tube and there is a linear increase in the normal reaction force with a defined slope (equal to the wall stiffness). Region II defines the transition zone where the reaction force starts increasing from zero as the beam comes in contact with the inner surface of the tube. $C^{1}$ continuity, that is, continuity of the force and the tangent stiffness, is considered for the derivation of the fitting polynomial in the transition zone.

Between $a$ and $b$ the reaction force due to the wall stiffness increases according to a second order polynomial (Fig. 6). Similarly, the reaction force due to wall damping increases according to a third order polynomial in the transition zone. The damping force is zero in Region I. There is linear damping in Region III.

Therefore, the net normal reaction force $F_{n}$, depending on the normal displacement $x_{n}$, and the normal velocity $v_{n}$, is given by

$$
F_{n}= \begin{cases}0 & \text { if } x_{n}<a \\ -(k / 2)(b-a) \xi^{2}-c_{w}(3-2 \xi) \xi^{2} v_{n} & \text { if } a \leq x_{n} \leq b \\ -k(b-a)(\xi-1 / 2)-c_{w} v_{n} & \text { if } x_{n}>b\end{cases}
$$

where $\xi$ is dimensionless parameter defined as $\xi=\left(x_{n}-a\right) /(b-a), \quad k$ is the wall stiffness, and $c_{w}$ the wall damping coefficient. $v_{n}$ is the velocity in the normal direction.

Friction Force A static friction model, in which the friction force depends on the normal force and the relative velocity only, is used for the calculation of friction forces. The friction model is based on the Coulomb model and has a continuous dependence on the sliding velocity near to zero sliding velocity region,

$$
\begin{aligned}
& F_{c}=-\mu F_{n} \\
& F_{t}=F_{c} \tanh \left(c_{v} v_{t}\right)
\end{aligned}
$$

where $\mu$ is the coefficient of friction between the contacting surfaces. $F_{n}$ is the normal reaction force acting at the node (Eqn. (12)). Eqn. (13a) gives the Coulomb friction. Eqn. (13b) is the continuous model, where $c_{v}$ is the velocity coefficient which determines the width of the transition region at $v_{t}=0$. Eqn. (13b) is used in the simulation as the friction force converges quickly to the Coulomb friction for increasing sliding speeds. A friction force always acts in the opposite direction of the motion on the tangent plane at the point of contact.

A Stribeck based continuous friction model [7] with increased friction at low velocity can also be implemented. This model can give us more accurate results in terms of friction behaviour, but at the expense of computational time.

Resultant force acting at the node The resultant force acting at the interacting node is given by:

$$
\mathbf{F}_{t o t}=F_{n} \hat{\mathbf{e}}_{n}+F_{t} \hat{\mathbf{e}}_{t}
$$

where $F_{n}$ and $F_{t}$ are the normal and tangential forces. $\hat{\mathbf{e}}_{n}$ is the unit vector along the normal direction and $\hat{\mathbf{e}}_{t}$ is the unit vector along the tangent.

\section{SIMULATION}

Simulation can provide insight into instrument behaviour during its insertion and fine manipulation thereafter. We are interested in the following attributes of the instrument:

- Force exerted on the instrument by the tube wall

- Maximum stress developed in the instrument

- Motion transmission at the tip

- Force transmission at the tip

The first two quantities will affect the life time of the instrument. Higher forces exerted on the instrument can lead to higher frictional wear. The maximum stress level provides a guideline to the instrument designers. Motion and force transmission characteristics are important for control purposes. Motion hysteresis can give rise to many control problems. Force transmission is important when the instrument tip is interacting with the tissues.

The following parameters are chosen as variables for the simulation: 
TABLE 1. MECHANICAL PROPERTIES

\begin{tabular}{lllll}
\hline Mechanical Property & Unit & Stainless steel & Kevlar $^{\circledR}$ & Nylon \\
\hline Density, $\rho$ & $\left(10^{3} \mathrm{~kg} / \mathrm{m}^{3}\right)$ & 7.8 & 1.44 & 1.04 \\
Modulus of elasticity, $E$ & $\left(10^{9} \mathrm{~N} / \mathrm{m}^{2}\right)$ & 200 & 80 & 3 \\
\hline
\end{tabular}

TABLE 2. PARAMETERS FOR FEM CALCULATIONS

\begin{tabular}{lllll}
\hline Parameter & Unit & SS wire & Kevlar $^{\circledR}$ wire & Nylon wire \\
\hline Diameter & $\mathrm{mm}$ & 0.50 & 0.50 & 0.50 \\
Mass/length & $\left(10^{-3} \mathrm{~kg} / \mathrm{m}\right)$ & 1.53 & 0.283 & 0.204 \\
Rotational inertia/length & $\left(10^{-11} \mathrm{~kg} \cdot \mathrm{m}^{2} / \mathrm{m}\right)$ & 2.39 & 0.442 & 0.319 \\
Axial stiffness, $E A$ & $\left(10^{3} \mathrm{~N}\right)$ & 39.3 & 15.7 & 0.589 \\
Bending rigidity, $E I$ & $\left(10^{-6} \mathrm{~N} \cdot \mathrm{m}^{2}\right)$ & 614 & 245 & 9.20 \\
\hline
\end{tabular}

- Stiffness of the instrument

- Friction between the instrument and the tube

- Shape of the tube

- Clearance in the tube

We chose three different materials - stainless steel (SS), Kevlar ${ }^{\circledR}$, and nylon wire - for the study. They can cover different stiffness ranges for the instrument. The material properties are given in Tab. 1 [8,9]. The shape of the tube can be varied by choosing different shapes for the centreline defining the tube. Circular arcs of $90^{\circ}$ and Bézier curves are used for the simulation. Different friction values $(\mu=0.2,0.5$, and1.0) are used for the various simulations. Clearance in the tube can have a large influence on the instrument behaviour. However, the effect of clearance is not studied in this paper. The clearance can be fixed for the most practical cases and it is minimal. The clearance in all the simulations are fixed and exaggerated to provide more insight.

In SPACAR, the mechanical properties are defined with the following input parameters:

- Mass per unit length

- Axial stiffness, EA

- Bending rigidity, $E I$

We chose a wire of diameter $0.5 \mathrm{~mm}$. Input parameters are calculated accordingly for the different materials. They are shown in Tab. 2.
TABLE 3. PARAMETERS FOR SIMULATION

\begin{tabular}{lll}
\hline Parameters & Units & Values \\
\hline Length of the instrument & $\mathrm{mm}$ & 1000 \\
Number of elements & & 10 \\
Diameter of the tube, $D_{a}$ & $\mathrm{~mm}$ & 4.0 \\
Diameter of the tube, $D_{b}$ & $\mathrm{~mm}$ & 5.0 \\
Radius of circular arc & $\mathrm{mm}$ & 500 \\
\hline
\end{tabular}

\section{Simulation of Flexible Beam Insertion}

Simulation of the insertion of the flexible instrument is carried out in SPACAR as shown in Fig. 1. The instrument is constrained in rotation at the proximal end and the input motion is applied at the node along the axial direction, i.e. the $x$ axis. A constant linear velocity, $0.010 \mathrm{~m} / \mathrm{s}$, is applied till the entire length of the instrument is inside the tube. A constant acceleration, $0.010 \mathrm{~m} / \mathrm{s}^{2}$, is applied in the beginning. Similarly, a constant deceleration, $0.010 \mathrm{~m} / \mathrm{s}^{2}$, is applied at the end, so that the initial and final velocities are zero. The simulation parameters are given in Tab. 3 .

The shape of the tube is defined by an arc of $90^{\circ}$ with straight sections at both ends of the arc. This ensures that the instrument does not buckle under the influence of interacting forces in the beginning of the insertion.

Figure 7 shows the plot of forces acting at the first two 


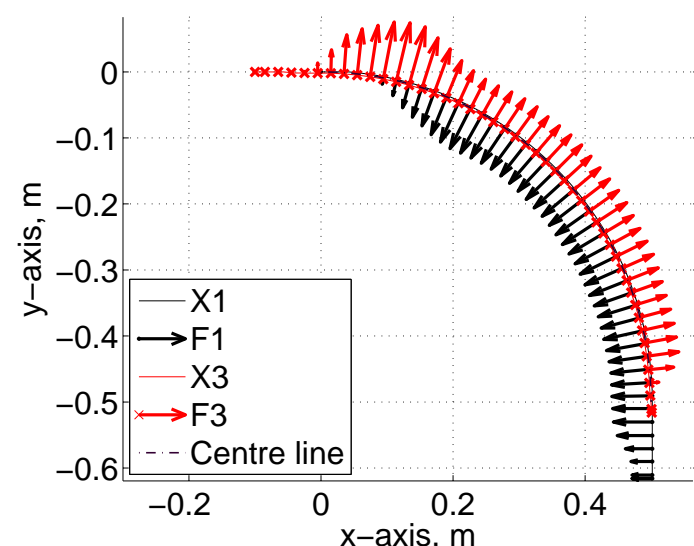

FIGURE 7. FORCES EXERTED AT NODES WHILE INSERTING IN CIRCULAR TUBE $(\mu=0.0)$

translational nodes at the distal end when the instrument is inserted into the tube. Only the first two translational nodes are considered for the sake of clarity, though similar plots can be obtained for all the nodes. The friction is assumed to be zero. A stainless steel wire of diameter $0.5 \mathrm{~mm}$ is considered for the instrument. $X 1$ and $X 3$ are the nodal positions of the first two translational nodes. $F 1$ and $F 3$ are the total interaction force acting at the respective nodes. It can be observed that the end node is making contact with the outer wall of the tube all the time, whereas the penultimate node is making contact with the inner wall as expected. Figure 8 shows the magnitude of forces acting at the first four distal nodes. The penultimate node experiences a larger force in the beginning when the end node started making contact with the tube. As the instrument advances further into the tube, the first two distal nodes experience larger forces compared to other nodes.

Similarly, a tube can be also defined using a Bézier curve. Figure 9 shows the plot of forces acting at the first two distal nodes when the instrument is inserted into the tube. Straight sections are defined at the entry and exit of the tube. The friction is assumed to be zero. A stainless steel wire of diameter $0.5 \mathrm{~mm}$ is considered for the instrument. The end node is experiencing a larger force as it goes through the first bend and then slowly decreases as the tube straightens. As the node advances further into the second bend, it experiences larger forces again. It can be observed that it is making contact with the concave surface, i.e. the outer wall of the tube. The penultimate node makes contact with the inner wall of the tube and the force acting at the node varies in a similar fashion. Figure 10 shows the magnitude of forces acting at the first four distal nodes as it moves inside the tube. The magnitude of forces acting at the various nodes are higher at the penultimate node. Figure 11 shows the forces acting at the various nodes after the complete insertion. The nodes

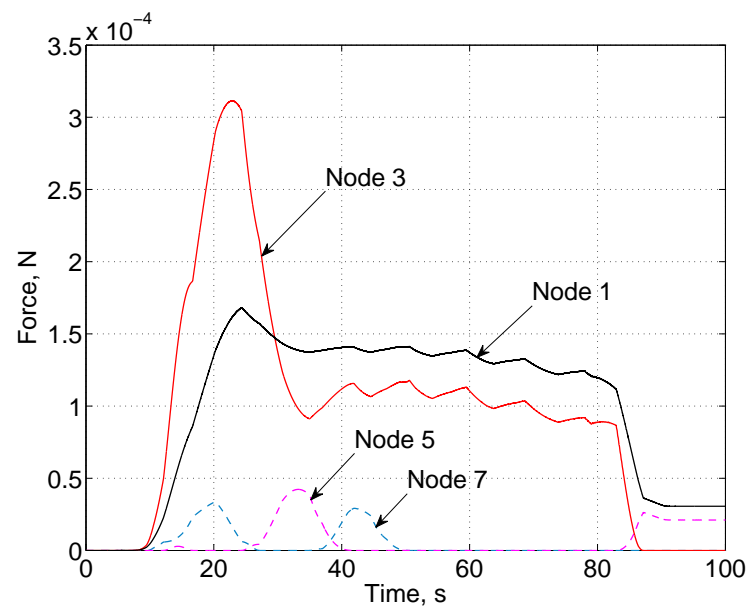

FIGURE 8. FORCES EXERTED AT NODES WHILE INSERTING IN CIRCULAR TUBE $(\mu=0.0)$

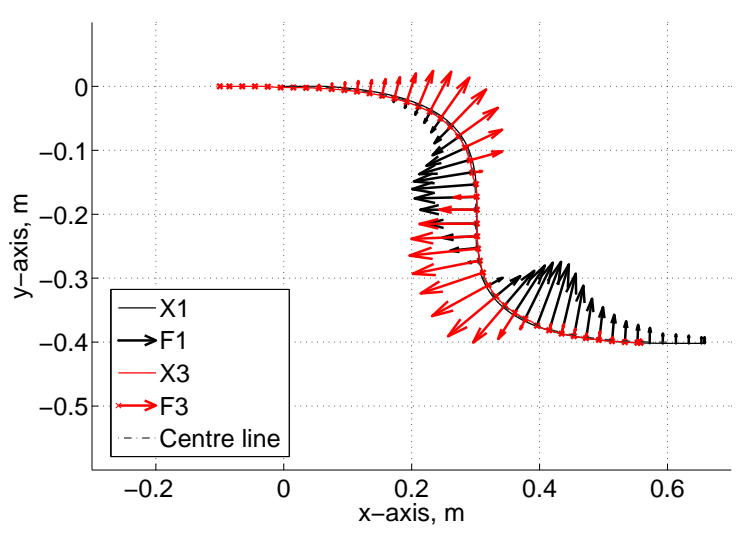

FIGURE 9. FORCES EXERTED AT NODES WHILE INSERTING IN A BÉZIER TUBE $(\mu=0.0)$

which are inside the bend experience larger forces as compared to the others. Node 1 is the distal end.

The total force exerted on the instrument while inserting in the tube can also be calculated. This will be equal to the total force exerted by the wall at individual nodes. Figure 12 shows the plot of the total force exerted by the wall on the instrument as it advances through the circular tube. There is no friction inside the tube. Forces exerted in $x$ - and $y$-directions are shown in the figure. Figure 13 shows the plot of the total force exerted on the instrument in a circular tube for $\mu=0.5$. Forces exerted in $x$ and $y$-directions are shown in the figure. When the instrument is advancing through the tube, the force exerted on the instrument is much higher when there is a friction inside the tube. The friction 


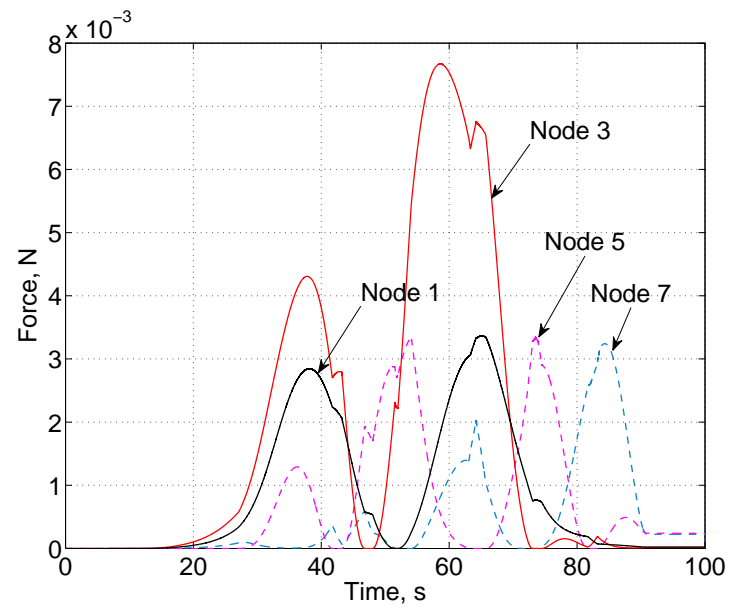

FIGURE 10. FORCES EXERTED AT NODES WHILE INSERTING IN A BÉZIER TUBE $(\mu=0.0)$

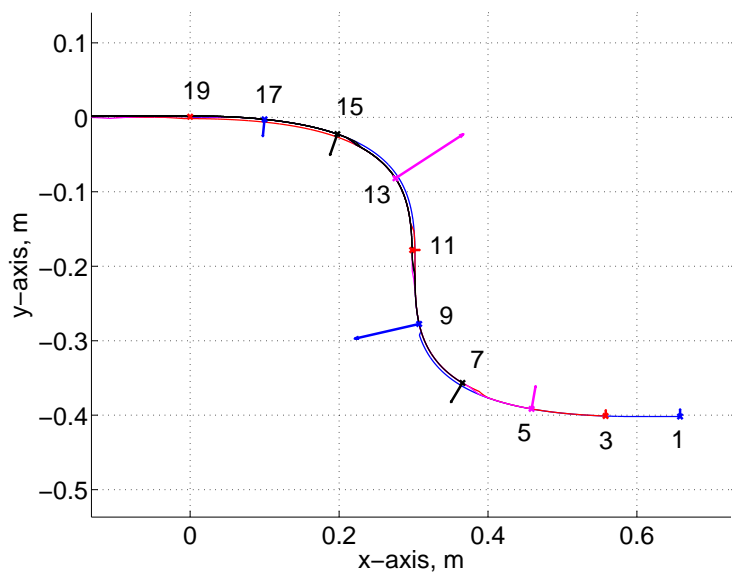

FIGURE 11. FORCES EXERTED AT VARIOUS NODES AFTER INSERTION IN A BÉZIER TUBE $(\mu=0.0)$

value has great influence when the instrument is moving inside the tube.

The shape of the tube will have influence on the insertion force too. Figure 14 shows the plot of the total force exerted on the instrument as it advances through a Bézier tube. In this case, there is no friction inside the tube.

\section{Simulation of Fine Manipulation}

Simulation of fine manipulation is carried out by firstly inserting the instrument completely inside the tube and then manipulating the tip by applying a small stroke input motion to

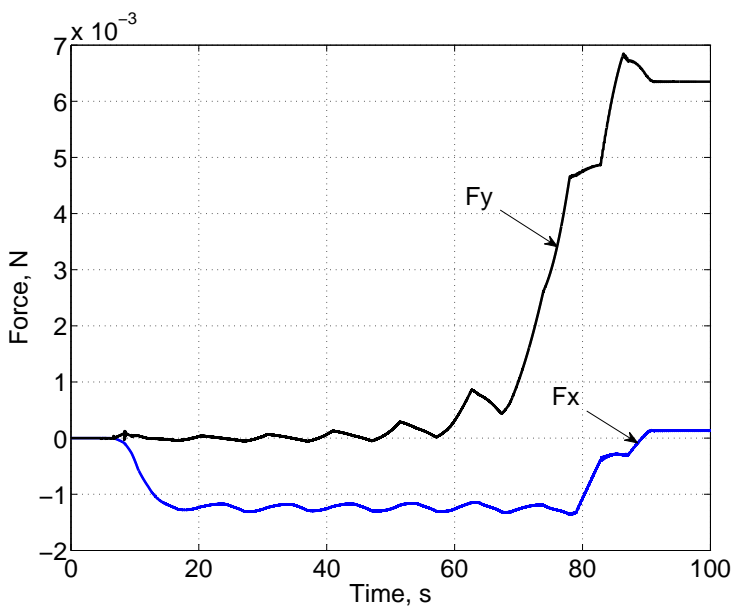

FIGURE 12. TOTAL FORCE EXERTED ON INSTRUMENT WHILE INSERTING IN A CIRCULAR TUBE $(\mu=0.0)$

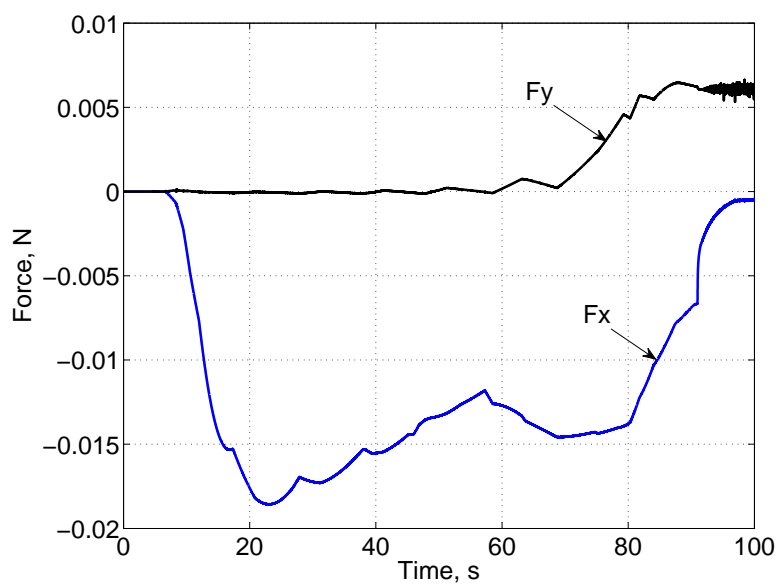

FIGURE 13. TOTAL FORCE EXERTED ON INSTRUMENT WHILE INSERTING IN A CIRCULAR TUBE $(\mu=0.5)$

the proximal end. The sine input motion is applied as follows:

$$
\begin{aligned}
x(t) & =A \sin (\omega t), \\
\dot{x}(t) & =\omega A \cos (\omega t), \\
\ddot{x}(t) & =-\omega^{2} A \sin (\omega t),
\end{aligned}
$$

where $A$ is the amplitude of motion, and $\omega=2 \pi f_{n}$. Here, the amplitude is chosen as $A=10 \mathrm{~mm}$ and the frequency, $f_{n}$, as $1 \mathrm{~Hz} . \quad x(t), \dot{x}(t)$, and $\ddot{x}(t)$ are the displacement, velocity, and acceleration applied at the input node, respectively. In the present work, we are only considering the unloaded condition. There is no load applied at the instrument tip. 


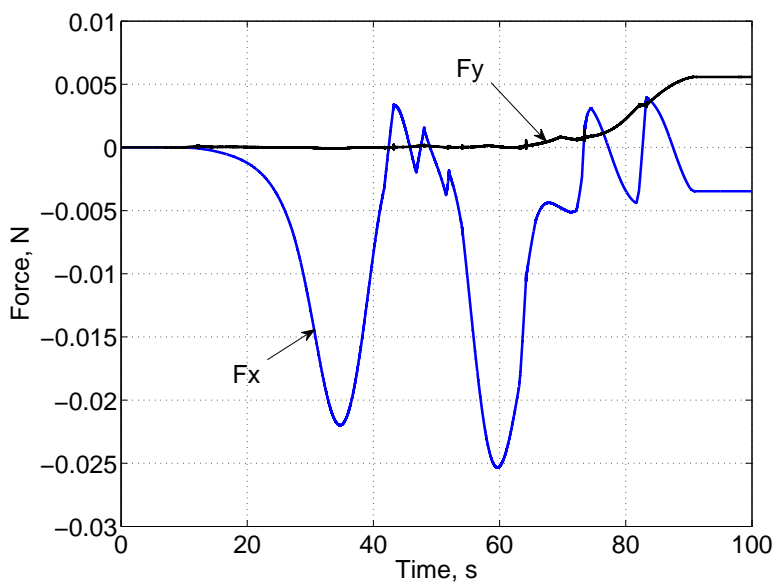

FIGURE 14. TOTAL FORCE EXERTED ON INSTRUMENT WHILE INSERTING IN A BÉZIER TUBE $(\mu=0.0)$

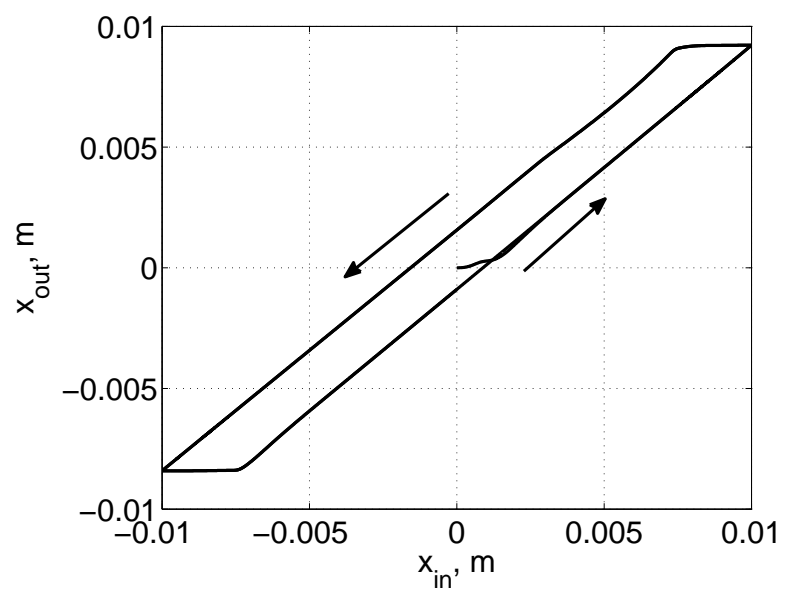

FIGURE 15. MOTION HYSTERESIS IN CASE OF CIRCULAR TUBE $(\mu=0.2)$

A stainless steel wire of diameter $0.5 \mathrm{~mm}$ is manipulated inside a circular tube. The coefficient of friction, $\mu$, is 0.2 . Figure 15 shows the plot of motion hysteresis. Displacement of the end node along the translation axis is plotted against the input displacement. Figure 16 shows the comparison of the translation velocity of the output and input node. The instantaneous velocity of the end node along the translation axis is plotted against the input velocity. Stick-slip bahaviour can be observed from the figure. This can lead to sudden movements and it can be very difficult to precisely control the tip motion.

Effect of Friction on Motion Hysteresis In order to understand the influence of the friction parameter on motion

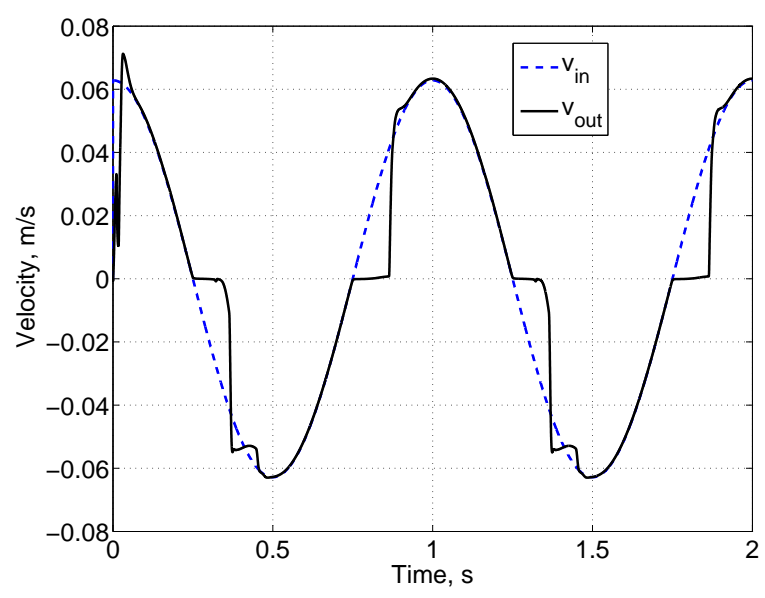

FIGURE 16. COMPARISON OF TRANSLATION VELOCITY AT INPUT AND OUTPUT $(\mu=0.2)$

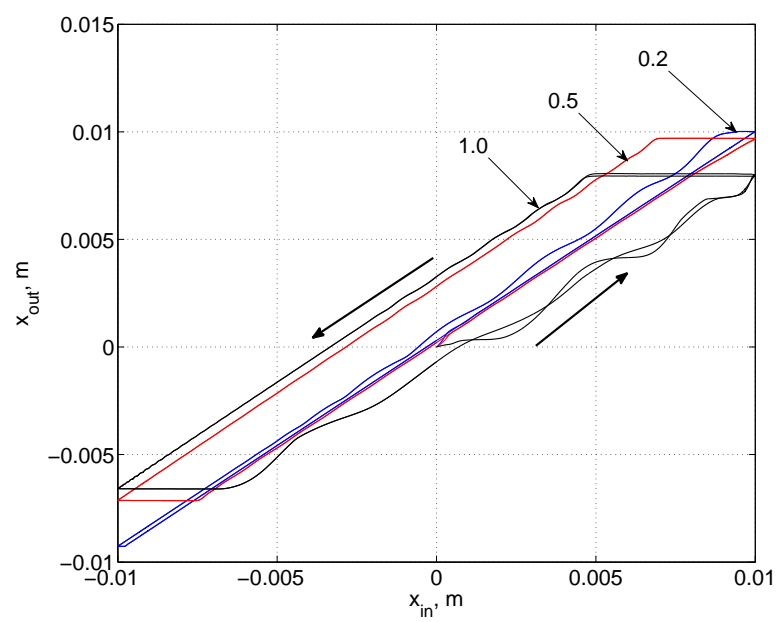

FIGURE 17. EFFECT OF FRICTION ON MOTION HYSTERESIS IN A BÉZIER TUBE FOR DIFFERENT COEFFICIENT OF FRICTION

hysteresis, a stainless steel wire of $0.5 \mathrm{~mm}$ diameter is manipulated inside the Bézier tube with different coefficients of friction $(\mu=0.2,0.5$, and1.0). The displacement of the end node along the translation axis is plotted against the input displacement for each case. Figure 17 shows the effect of friction on motion hysteresis. Friction has a large influence on the motion hysteresis. The backlash is larger and even unpredictable as the friction increases.

Effect of Bending Rigidity on Motion Hysteresis The bending rigidity of the instrument can have similar impact 


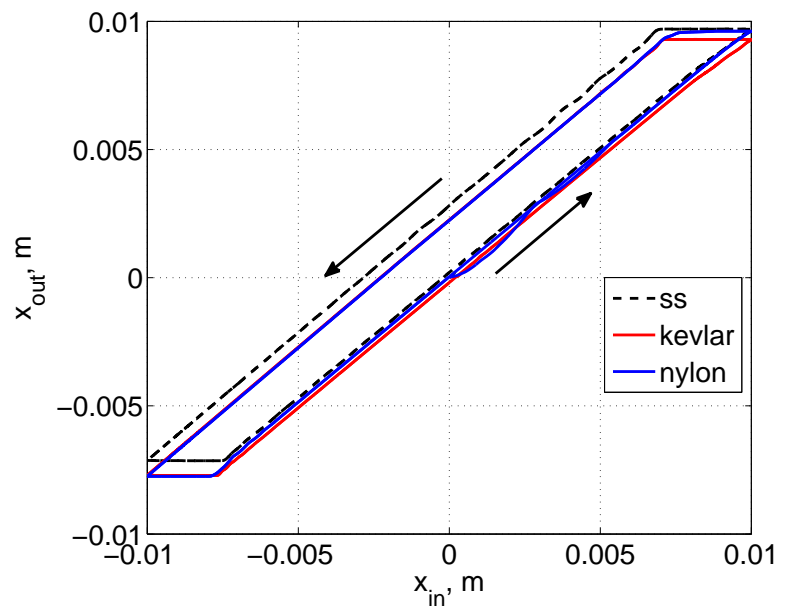

FIGURE 18. EFFECT OF BENDING RIGIDITY ON MOTION HYSTERESIS $(\mu=0.5)$

on the motion hysteresis. In this case, the coefficient of friction, $\mu$, is fixed at a value of 0.5 . Stainless steel, Kevlar ${ }^{\circledR}$, and Nylon wires of $0.5 \mathrm{~mm}$ diameter are used for the simulation. Again, the displacement of the end node is compared with the input displacement for each case. Figure 18 shows the influence of the bending rigidity on motion hysteresis. All the three plots show similar variation. There is no significant difference observed in the three plots. This can be expected as the virtual backlash due to friction depends on the normal reaction force and stiffness [10]. In this case both the friction force caused by the normal reaction force, and the driving stiffness of the instrument are directly proportional to the Young's modulus. Therefore the stiffness has no influence on the virtual backlash.

\section{CONCLUSION}

A planar model of a flexible instrument inside a tube has been set up. A Stribeck based continuous friction model has been implemented. The forces exerted on the instrument because of the interaction with the tube can be calculated. The motion hysteresis and stick-slip behaviour have been observed in the simulations. The friction, bending rigidity of the instrument, and the shape of the tube can have a large influence on the performance of the instrument and ultimately on its control. The modelling and simulation results can provide some estimation and guidelines to the designers and control engineers to design and control a robust flexible endoscopic surgical system. Future work aims at the validation of the simulation results with experimental results. In addition a spatial model will be set up to simulate rotational behaviour. Loading at the instrument tip can affect the instrument behaviour significantly, which will be undertaken in the future work.

\section{ACKNOWLEDGMENT}

This research is a part of the TeleFLEX project, which is funded by the Dutch Ministry of Economic Affairs and the Province of Overijssel, within the Pieken in de Delta (PIDON) initiative.

\section{REFERENCES}

[1] Camarillo, D. B., Krummel, T. M., and Salisbury, J. K., 2004. "Robotic technology in surgery: Past, present, and future". The American Journal of Surgery, 188(Supplement 1), pp. 2-15.

[2] Jonker, J. B., and Meijaard, J. P., 1990. Multibody Systems Handbook. Springer-Verlag, Berlin, ch. SPACAR - computer program for dynamic analysis of flexible spatial mechanisms and manipulators, pp. 123-143.

[3] Werff, K. v. d., and Jonker, J. B., 1984. "Dynamics of flexible mechanisms". In Computer aided analysis and optimization of mechanical system dynamics, E. J. Haug, ed., NATO ASI series. Series F ; vol. 9, Springer-Verlag, pp. 381-400.

[4] Aarts, R. G. K. M., and Jonker, J. B., 2002. "Dynamic simulation of planar flexible link manipulators using adaptive modal integration". Multibody System Dynamics, 7(1), pp. 31-50.

[5] Dewey, B. R., 1988. Computer Graphics for Engineers. Harper \& Row, Publishers, New York.

[6] Hoff, H. t., 1993. "Scanning mechanisms for intravascular ultrasound imaging: a flexible approach". $\mathrm{PhD}$ thesis, Erasmus Universiteit Rotterdam, the Netherlands.

[7] Andersson, S., Söderberg, A., and Björklund, S., 2007. "Friction models for sliding dry, boundary and mixed lubricated contacts". Tribology International, 40(4), pp. 580 - 587. NORDTRIB 2004.

[8] Gere, J. M., and Timoshenko, S. P., 1999. Mechanics of MATERIALS, 4th SI ed. Stanley Thornes (Publishers) Ltd, United Kingdom.

[9] - 2011. KEVLAR ${ }^{\circledR}$ technical guide. On the WWW, May. URL http: / / www2 . dupont. com.

[10] Soemers, H. M. J. R., 2010. Design Principles for Precision Mechanisms. T-Point Print. ISBN 978-90-365-3103-0. 